\title{
Evaluación de la fuerza de adhesión de pegamentos para papel
}

\section{Evaluation of adhesive strength of paper glues}

\author{
Aguilar Jaqueline ${ }^{1}$, Benzadón María ${ }^{1}$, De León Erick ${ }^{1 *}$, Sánchez María ${ }^{1}$, Maritza Cedeño ${ }^{2}$ \\ ${ }^{1}$ Licenciatura en Ingeniería Industrial - Centro Regional de Azuero - Universidad Tecnológica de \\ Panamá ${ }^{2}$ Docente de Pregrado-Centro Regional de Azuero-Universidad Tecnología de Panamá \\ Grupo de Investigación de Azuero en el Área Empresarial
}

\begin{abstract}
Resumen En esta investigación se utilizó el diseño experimental para determinar si existe o no diferencia significativa en la fuerza de adhesión de cuatro tipos de pegamentos, mediante un diseño de bloques completos al azar, evaluando los resultados mediante análisis de varianza, identificando los valores obtenidos con la distribución de Fisher y verificando si se aceptan o no las hipótesis. La hipótesis nula para tratamiento se acepta, lo que significa que al 5\% de significancia podemos concluir que la fuerza adhesiva de las cuatro marcas de pegamento es igual. En el análisis de los bloques se encontró que los bloques (tipo de papel) no tiene un efecto significante en el diseño experimental.
\end{abstract}

Palabras clave Adhesivo, fuerza de adhesión, diseño experimental, diseño de bloque, calidad.

\begin{abstract}
In this research, design of experiment methodology was used to determine whether or not there are significant difference in the adhesive force of four types of adhesives. with a design of a randomized complete block, evaluating the results through analysis of variance, checking values obtained with the Fisher's distribution and verifying whether or not to accept the null hypothesis. All this is done to obtain valuable information when buying adhesives and verify quality.
\end{abstract}

Keywords Ahesive, adhesive force, design of experiments, block design, quality.

*Corresponding author: erick.deleon1 @utp.ac.pa

\section{Introducción}

A inicios del siglo XX, las empresas vivían una crisis de escases de recursos, baja productividad y deficiencias en la calidad, todo esto a causa de la primera guerra mundial. Era necesario entonces, volver más eficientes las operaciones e identificar las variables que intervienen al momento de desarrollar un proceso. Para ello el estadístico Ronald Fisher desarrolla las bases del diseño experimental, las cuales se basan en el análisis de varianza y la aleatorización.

El diseño de experimentos permite analizar datos mediante modelos estadísticos para observar la interacción entre las variables independientes y cómo afectan a la variable dependiente [1]. Es por esto que el diseño experimental es parte fundamental de la experimentación y su objetivo es validar o rechazar las hipótesis que se plantean durante una investigación. Así el diseño de experimentos representa una de las mejores herramientas para analizar datos obtenidos en diferentes condiciones (variables independientes) propuestas por el investigador, verificando cuáles de estas variables intervienen de manera significativa en la reacción o respuesta medida. La aleatorización es un componente importante para el diseño experimental, puesto que asigna al azar unidades experimentales para cada prueba, garantizando así la independencia de las observaciones. En un experimento también es importante el control local, el cual consiste en hacer variar solo los factores que se van a estudiar en el experimento, dejando constantes los demás factores; en la vida real, el control local es difícil de lograr ya que existen algunas variables incontrolables que pueden afectar el resultado. Lo anterior introduce el concepto de error experimental, el cual mide las variaciones de las observaciones hechas en condiciones supuestamente idénticas [2]. El análisis de varianza es la herramienta utilizada para medir la variación de más de dos medias o resultados obtenidos en un experimento, comprobando según la distribución de Fisher, si se acepta o se rechaza la hipótesis nula, cuyo enunciado consiste en determinar si existe o no, diferencia significativa entre los valores observados.

\section{Enunciado del problema}

Se desea determinar si existe diferencia significativa entre la fuerza de cohesión de las diferentes marcas de los adhesivos para papel. Se piensa que la superficie de pegado influye sobre los resultados, por lo que se realizó un bloqueo con los tipos de papel comúnmente utilizados: papel de construcción, cartoncillo y cartulina. 


\section{Justificación}

La historia de los pegamentos como ciencia, tecnología y medio para unir materiales se encuentra desde los primeros vestigios de los hombres de la prehistoria. Durante esta época se utilizaban pegamentos y adhesivos de origen natural para la creación de pinturas, así como para la fabricación de armas y utensilios. Durante un largo periodo de la historia antigua los pegamentos eran conocidos y utilizados por las diversas culturas y civilizaciones, tales como los sumerios, babilonios, egipcios, chinos, entre otros; utilizaron los adhesivos en numerosas edificaciones y construcciones de objetos de diversa índole [3]. Actualmente las personas y empresas buscan el mejor producto para pegar diversos materiales. Sin embargo, la calidad de un pegamento se relaciona con las especificaciones o el precio. Estos productos son una parte fundamental del mundo de las manualidades; saber elegir el correcto en cada caso puede ocasionar contratiempos y costos, es por ello que desarrollamos este estudio con la finalidad de brindar más información sobre la calidad de los pegamentos.

Se medió la fuerza de adhesión de los tipos de pegamentos, el cual es un requisito de funcionalidad en las especificaciones de calidad; de esta manera se brinda información que sirva de apoyo a personas y empresas, al momento de seleccionar un pegamento que cumpla con las características preestablecidas [4].

\section{Diseño experimental}

\subsection{Unidades experimentales}

Para el experimento propuesto se utilizó como unidad experimental, trozos o muestras de tamaño $3 \times 2$ ", de papel de construcción, cartulina y cartoncillo.

\subsection{Factor, niveles y riesgo}

Las variables o factores que se identifican para este experimento son: tipo de adhesivo, tipo de papel, cantidad de adhesivo aplicado, área de aplicación, tiempo de secado del adhesivo, procedimiento de lectura. Se escoge como factor de interés o tratamiento, el tipo de adhesivo. La variable o factor, tipo de papel se utiliza para la definición del tipo de diseño experimental. El resto de las variables se controlan en el experimento de la siguiente forma:

- Cantidad de adhesivo: Se utilizó la misma cantidad de adhesivo en cada prueba.

- Área de aplicación: Se definió la misma área de aplicación en cada unidad experimental.

- Tiempo de secado: Se tomó igual tiempo de secado en cada réplica del experimento.

- Procedimiento de lectura: Todas las réplicas fueron grabadas para leer con exactitud de la pantalla de grabación, la lectura del dinamómetro.

El nivel de riesgo de esta investigación, se definió con un $5 \%$, el cual representa el valor del Nivel de Significancia.

\subsection{Variable dependiente e independiente}

Se define la variable independiente como aquella que se manipula y se hace variar en el experimento, en este caso, los diferentes tipos de adhesivos. Se definen cuatro niveles para la variable representadas por las marcas de los adhesivos denominadas por las letras A, B, C y D. La variable dependiente es la que se mide y se observa en la investigación, siendo esta la fuerza de adhesión.

\subsection{Elección del diseño experimental}

Se utilizó el diseño de bloques completo al azar, porque existe un factor que puede influir en el resultado del experimento. En este caso se prevé que la fuerza de adhesión de los diferentes tipos de adhesivos puede estar influido por los tipos de papel en donde se aplican todos los tratamientos a los bloques para eliminar sesgos en la investigación y errores en los resultados. El modelo matemático para este diseño es: [2]

$$
y_{i j}=\mu+\tau_{i}+\beta_{j}+\delta_{i j}\left\{\begin{array}{l}
i=1,2, \ldots, a \\
j=1,2, \ldots, b
\end{array}\right.
$$

Las hipótesis que se desean comprobar en este estudio son las siguientes:

Tratamiento: Tipo de adhesivo.

$\mathrm{H}_{\mathrm{o}}$ : No existe diferencia significativa entre la fuerza de adhesión de los diferentes tipos de adhesivos.

Bloques: Tipos de papel

$\mathrm{H}_{0}$ : El tipo de papel no causa un efecto significante en el diseño experimental.

\section{Materiales y Métodos}

\subsection{Materiales}

El experimento se desarrolló utilizando los siguientes materiales: papel de construcción, cartulina y cartoncillo; cuatro tipos de pegamentos de diferentes marcas, en donde asignamos las siguientes letras para los mismos, A: Elmer's B: Pritt, C: Crayola, D: Faber Castell. Cabe señalar que los pegamentos cuentan con especificaciones de uso y calidad similares. Para evaluar la fuerza de adhesión se utilizó un dinamómetro con una escala de 20 Newton [5]; tape para sujetar el papel al dinamómetro, tijeras y goteros.

\subsection{Metodología}

El procedimiento para realizar el análisis y medir la fuerza de adhesión de pegamentos se desarrolla de la siguiente manera:

a. Recortar los tipos de papel con una dimensión de 3x2". Para este caso se selecciona cuatro unidades experimentales por bloque para desarrollar 12 réplicas del experimento.

b. Aleatorizar las unidades experimentales por bloque (primer dígito del código de la tabla 1). 


\begin{tabular}{|c|c|c|c|c|}
\hline Tabla 1. Esquema de aleatorización \\
\hline Bloques & A & B & C & D \\
\hline Papel de construcción & $4 \mathrm{~A} 9$ & $2 \mathrm{~B} 12$ & $3 \mathrm{C} 10$ & $1 \mathrm{D} 1$ \\
\hline Cartulina & $4 \mathrm{~A} 5$ & $1 \mathrm{~B} 2$ & $4 \mathrm{C} 3$ & $3 \mathrm{D} 8$ \\
\hline Cartoncillo & $3 \mathrm{~A} 11$ & $3 \mathrm{~B} 4$ & $2 \mathrm{C} 7$ & $4 \mathrm{D} 6$ \\
\hline
\end{tabular}

En el código 4C3, de la tabla 1, el primer número corresponde a la unidad experimental número 4, en dónde; $\mathrm{C}$ corresponde a la marca de pegamento Crayola (Washable School Glue).

c. Asignar tratamientos aleatoriamente dentro de cada bloque (Segunda posición en el código, tabla 1).

d. Aleatorizar el orden de las pruebas (tercer dígito en tabla 1). En el código 4C3, el número tres significa que el tratamiento $\mathrm{C}$, a la unidad experimental 4 , se aplicará en tercer lugar, en cuanto al ordenamiento de las pruebas.

e. Aplicar con el uso de un gotero 3 gotas de pegamento de la marca correspondiente sobre la unidad experimental como se observa en la figura 1.

f. Unir los dos extremos del papel sobre el cual se aplicó la goma. Cabe señalar que el área de pegado es de aproximadamente dos centímetros cuadrados.

g. Esperar cinco minutos para el secado de la goma.

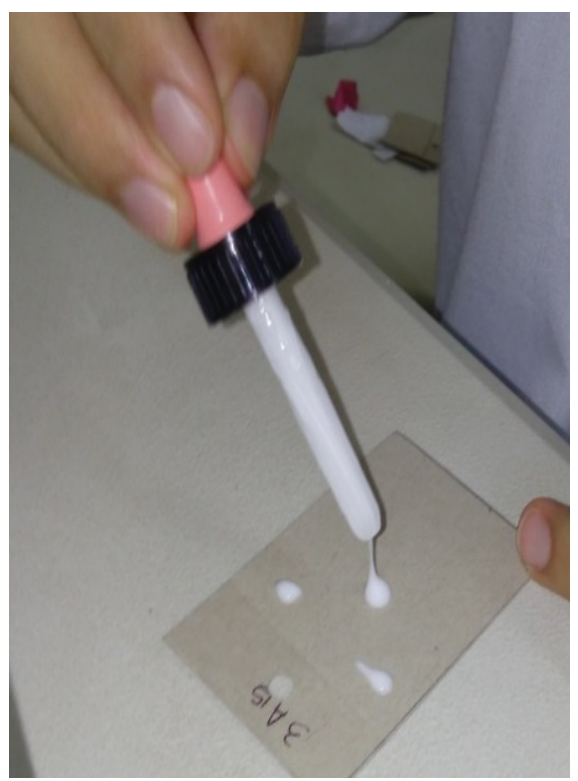

Figura 1. Aplicación de pegamento a la unidad experimental.

h. Colocar la unidad experimental sobre el gancho o extremo del dinamómetro y halar hasta la ruptura de la muestra, como se observa en la figura 2.

i. Tomar la medida de fuerza en el dinamómetro, justo en el momento de la ruptura.

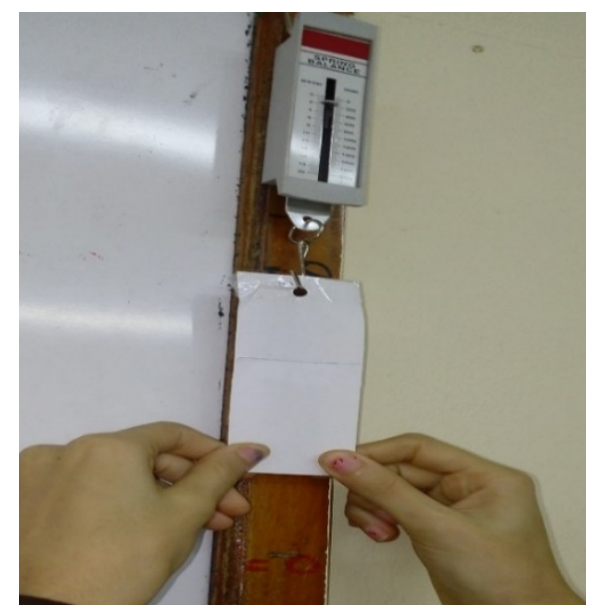

Figura 2. Halar la unidad experimental para medir mediante dinamómetro la fuerza de adhesión.

\section{Resultados}

Siguiendo el orden de las pruebas y el procedimiento, en la tabla 2 se observan los resultados de las mediciones:

Tabla 2. Fuerza de adhesión en newton (n)

\begin{tabular}{|c|c|c|c|c|}
\hline Marca & A & B & C & D \\
\hline Papel de construcción & 10 & 21 & 3 & 1.5 \\
\hline Cartulina & 6.2 & 9 & 7 & 8.2 \\
\hline Cartoncillo & 21 & 18 & 8 & 21 \\
\hline
\end{tabular}

Mediante el software Minitab, se introducen los datos y se realiza el análisis de varianza, cuyos valores correspondientes a las fuentes de variación, grados de libertad, cuadrado medio y valor de $\mathrm{F}$ de Fisher, estos resultados se presentan en la tabla 3.

Tabla 3. Análisis de varianza: factor-tipos de adhesivos, bloque-tipo de papel

\begin{tabular}{|c|c|c|c|c|}
\hline Fuente & GL & SC & MC & F \\
\hline B-Papel & 2 & 196.485 & 98.2425 & 3.05 \\
\hline T-Adhesivo & 3 & 155.109 & 51.7031 & 1.60 \\
\hline Error & 6 & 193.468 & 32.2447 & \multicolumn{1}{|c}{} \\
\cline { 1 - 4 } Total & 11 & 545.063 & \multicolumn{2}{|c}{} \\
\cline { 1 - 3 } & &
\end{tabular}

Para analizar los resultados se comparan los valores de Fisher, obtenidas del cuadro de ANOVA (Analysis of Variance), con los valores críticos en una tabla de distribución de Fisher, al 5\% de significancia. En cuanto al Factor analizado (tipo de adhesivos), se observa que la $\mathrm{F}$ obtenida del análisis de varianza es de 1.60 , menor al valor crítico de $\mathrm{F}$ en la tabla de distribución de Fisher (3.59), al 5\% de significancia y 3 grados de libertad.

Para los bloques se observa que la F calculada es de 3.05, mientras que su valor en la distribución de Fisher es de 3.98, siendo el valor de F calculada menor al de la distribución 


\section{Conclusiones}

1. Para el desarrollo de un experimento, planeado estadísticamente, es preciso la definición clara de los elementos presentes, tales como, unidades experimentales, bloques, réplicas del experimento. Esto nos permite desarrollar un análisis de varianza para determinar si existe o no diferencia entre tratamientos y obtener información valiosa sobre el comportamiento de la variable de respuesta, al aplicarse cambios controlados en la variable independiente.

2. Por medio del DoE, en esta investigación se concluye que existe evidencia estadística, al 5\% de significancia, de que los pegamentos evaluados poseen iguales propiedades en cuanto a, fuerza de adhesión, por lo que cumplen su requisito de calidad primario, es decir; independientemente del pegamento que se utilice, se puede obtener lo que el cliente espera, lo cual es poder adherir adecuadamente las superficies.

3. El cliente o usuario final, puede confiar en seleccionar aquella marca de pegamento de menor costo, con la seguridad de obtener el resultado esperado.

4. No era necesario bloquear por tipo de papel en este experimento, por lo que, para futuros diseños experimentales, se propone realizarlo mediante un diseño completo al azar, utilizando como unidades experimentales cualquiera de los materiales utilizados en este experimento.

\section{RECONOCIMIENTO}

A la profesora Maritza Cedeño por guiarnos en la redacción de este artículo, al profesor Cenobio Villalobos por brindarnos asesoría en la generación de la idea de investigación y al profesor Secundino Villarreal por suministrarnos herramientas para realizar las mediciones correspondientes al diseño experimental.

\section{REFERENCIAS}

[1] Douglas, Montgomery, Diseño y Análisis de Experimentos, 2nd ed., México DF; Limusa Wiley, 2001.

[2] Humberto Gutiérrez P., Análisis de Experimentos, Guadalajara; Mc Graw Hill, 2008.

[3] Online available: http://www.losadhesivos.com/historiade-los-adhesivos.html

[4] Humberto Gutiérrez, Calidad Total y Productividad, tercera Edición, Mc Graw Hill, México, 2010.

[5] Mario Madrid, Tecnología de la Adhesión, España, Departamento técnico de LOCTITE. 